\title{
Diferenças entre géneros nas habilidades: correr, saltar, lançar e pontapear
}

\author{
Maria Isabel Mourão Carvalhal, José Vasconcelos-Raposo \\ Universidade de Trás-os-Montes e Alto Douro - UTAD, Vila Real, Portugal
}

Carvalhal, M.; Vasconcelos-Raposo, J.; Diferenças entre géneros nas habilidades: correr, saltar, lançar e pontapear. Motricidade 3(3): 44-56

\section{Resumo}

Fundamentação: o estudo tem como objectivo verificar diferenças entre géneros na prestação qualitativa e quantitativa nas habilidades locomotoras: correr, saltar e habilidades manipulativas: lançar, pontapear. Metodologia: O estudo integrou uma amostra de 141 crianças, 79 do sexo msculino e 62 do sexo feminino, de 7 e 8 anos de idade, do $1 .^{\circ}$ ciclo do ensino básico do distrito de Vila Real. O produto foi avaliado através da média das distâncias obtidas nos diferentes ensaios nas habilidades saltar, lançar e pontapear e o tempo médio obtido nos cinco ensaios da corrida. O processo foi avaliado com base na checklist de Gallahue ${ }^{10}$. Resultados: Quando comparamos a prestação entre rapazes e raparigas registamos diferenças estatisticamente significativas, quer relativas ao processo, quer ao produto de desempenho em todas as habilidades. Nas habilidades manipulativas as diferenças foram de maior magnitude, tendo o sexo masculino apresentado padrões de execução e resultados superiores aos do sexo feminino. Conclusões: Os desempenhos significativamente superiores apresentados pelos rapazes em todas as habilidades, nomeadamente no lançar e pontapear evidenciam a influência dos factores de ordem sociocultural no desenvolvimento e aprendizagem destas habilidades.

Palavra-chave: habilidades motoras, género, avaliação processo e produto.

\section{Abstract}

Differences between gendres in motor skills: running, jumping, throwing and kicking

Back Ground: the purpose of the present study was to compare the differences between boys and girls in the basic motor skills: throwing and kicking (process and product). Methodology: the sample consisted of 141 children, 79 boys and 62 girls aged between 7 and 8 who attended the second year of first phase of first cycle basic school in the district of Vila Real. The motor product was assessed by the mean distance obtained in repeated throws, jumps and kicks trials and time in running. The process was assessed with a Gallahue's ${ }^{10}$ checklist. Results: when we compared male and female motor performance significant effects were observed both at the level of motor process and in the product. Males showed highest product and process performance in manipulative skills like throwing and kicking. Conclusions: the higher levels of performance from males in all the skills, mannely in throwing and kicking showed the influence of sociocultural factors in motor learning skills.

Keywords: motor skills, gender, process and product assessment. 


\section{Introdução}

Apesar de, durante a infância, não se registarem diferenças na prestação motora entre os géneros, as diferenças vão acentuando-se, à medida que a idade avança, tornando-se significativas durante a adolescência $a^{7,16,17,18}$.

As teorias biológicas justificam as diferenças entre géneros na prestação motora com base nas variáveis antropométricas, como o tamanho corporal, morfologia e proporções corporais ${ }^{21,29} \mathrm{e}$ na hereditariedade ${ }^{6}$.

$\mathrm{Na}$ opinião de Malina ${ }^{23}$, um maior tamanho corporal e um maior comprimento de alavancas por parte do sexo masculino vai proporcionar-lhe um melhor desempenho nas tarefas grosseiras que exigem deslocamento do corpo no espaço e projecção de objectos, enquanto que uma maior quantidade de massa gorda, que equivale a mais peso morto para deslocar, por parte do sexo feminino, vai dificultar-lhe as tarefas de deslocação do corpo no espaço. Haubenstricker e Sapp ${ }^{15}$ defendem que as ligeiras diferenças verificadas a nível do tamanho e estrutura corporal entre os rapazes e raparigas, na infância, podem contribuir para as diferenças de prestação entre os dois sexos. A superioridade dos rapazes na altura e comprimento do braço, funcionam como vantagens a nível das alavancas na tarefa do lançamento, especialmente quando estas vantagens estão associadas com uma maior percentagem de massa magra e maiores oportunidades de prática, como é o caso dos elementos masculinos.

As teorias que explicam as diferenças com base nos factores do envolvimento centram-se nas variáveis sócio-culturais como: os factores educativos, as expectativas culturais e as oportunidades de prática ${ }^{20,11,39}$. Os papéis atribuídos a cada um dos sexos são diferentes, e desde que a criança nasce é estimulada a seguir um determinado modelo. Assim, as expectativas geradas para cada um dos géneros são diferentes, dependendo do modelo a seguir, sendo também dife- rentes as oportunidades de prática, e o encorajamento dado a cada um dos sexos. De acordo com esta perspectiva, as diferenças são socialmente induzidas, argumentando os defensores desta teoria, que se fossem dadas as mesmas oportunidades, o mesmo encorajamento e as mesmas expectativas, a rapazes e raparigas, as diferenças de prestação, antes da puberdade, seriam menores.

$\mathrm{Na}$ opinião de Thomas, Thomas e Gallagher ${ }^{42}$, as características do envolvimento (isto é, a situação social, a quantidade de exercício, os colegas, os pais, os professores, as oportunidades, o encorajamento e a prática), são os factores principais na produção das diferenças entre os sexos. As variáveis biológicas (maturação e gordura) parecem mediar estas diferenças.

\section{Habilidades Locomotoras: Corrida e Salto}

$\mathrm{Na}$ infância as diferenças na prestação da corrida entre géneros não se verifica ${ }^{28}$, ou são muito ligeiras ${ }^{24,25}$. Elas tornam-se mais evidentes a partir dos 9/10 anos e persistindo através da adolescência, devido à estabilização verificada entre as raparigas no período da adolescência ${ }^{22}$.

Regista-se uma melhoria durante a infância nos dois géneros, no entanto, a prestação das raparigas torna-se estável na adolescência, enquanto nos rapazes a prestação apresenta melhorias desde a infância e na adolescência ${ }^{19}$.

Quando comparamos rapazes e raparigas quanto ao padrão de execução da corrida não se registam diferenças significativas ${ }^{33,28}$, no entanto, os rapazes atingem o padrão maduro mais cedo e as raparigas levam mais tempo a fazer a passagem do estádio intermédio para o estádio maduro ${ }^{37}$.

$\mathrm{Na}$ habilidade saltar, na infância, embora os rapazes apresentem níveis de prestação superiores aos das raparigas ${ }^{25}$, as diferenças são pequenas ${ }^{27}$, tornando-se significativas durante o período da adolescência ${ }^{16}$. Embora ambos os géneros apre- 
sentem melhorias à medida que a idade avança, na infância, as melhorias são de maior magnitude nos rapazes do que nas raparigas ${ }^{24}$. A partir dos 14 anos, as raparigas apresentam decréscimos na prestação; os rapazes, pelo contrário, melhoram a sua prestação até aos $17 \operatorname{anos}^{16,25}$.

De acordo com Seefeldt e Haubenstricker ${ }^{16}$ (1982), no desenvolvimento da forma de saltar, não se registam diferenças entre os sexos, como acontece com outras habilidades (lançar, receber e sticar). As diferenças entre géneros a nível da forma de execução surgem depois dos 9 anos e tem a ver com o facto de os rapazes conseguirem, mais cedo do que as raparigas, flectir a coxa e o tronco, o que acontece logo a partir dos 9 anos até aos 12 anos'. Estes resultados levam-nos a concluir que os rapazes progridem mais cedo para o padrão maduro do salto, a julgar pelas diferenças verificadas na sequência da evolução deste padrão.

\section{Habilidades Manipulativas: Lançar e Pontapear}

O lançamento, comparativamente com a corrida e o salto é a habilidade onde se registam as maiores diferenças entre géneros. Antes da puberdade, o desempenho das raparigas na distância do lançamento atinge apenas $54 \%$ e $57 \%$ do dos rapazes, enquanto nas habilidades corrida e salto representa $96 \%$ e $90 \%$ respectivamente $^{14}$. É nesta habilidade que se verificam as maiores diferenças entre rapazes e raparigas, apresentando os rapazes desempenhos significativamente superiores aos das raparigas desde muito cedo na infância. Desde a infância, os rapazes lançam significativamente mais longe do que as raparigas ${ }^{5,44,28}$ e estas diferenças prolongam-se pela adolescência ${ }^{16,25,34}$. As diferenças são sempre significativas, quer quando a prestação foi avaliada tendo em conta a distância ou a velocidade $^{2,13}$.

As habilidades manipulativas como o lançamento e o pontapé são aquelas onde se registam as maiores diferenças e logo desde muito cedo na infância. Tal como nas outras habilidades estudadas, parece não haver justificação a nível biológico, para esta diferença entre os sexos, pelo que serão factores mais de ordem cultural, como o encorajamento e a prática, os responsáveis por estes resultados ${ }^{46}$. Por outro lado, Eckert ${ }^{7}$ defende que as diferenças se devem à maior força e um tamanho corporal superior por parte dos rapazes. Por outro lado, Thomas, Thomas e Gallagher ${ }^{42}$ e Thomas ${ }^{41}$ são de opinião que a magnitude das diferenças registada no lançamento pode ser reflexo de uma adaptação filogenética da espécie humana.

O Homem na caça e na pesca utilizava movimentos de lançar, o que lhe proporcionou a aquisição e melhoria de um padrão de lançar, contrariamente às mulheres que exerciam funções que não exigiam a projecção de objectos, como o tomar conta dos filhos e cozinhar.

Estes argumentos poderão ser postos em causa, face às conclusões obtidas num estudo recente sobre a utilização e fabricação de instrumentos em chimpanzés. O estudo verificou, que, ao contrário do que se pensava, as fêmeas chimpanzés e os chimpanzés imaturos apresentaram uma maior frequência de períodos de caça e de utilização de instrumentos comparativamente com os dos machos ${ }^{31}$.

Para os investigadores Thomas e French ${ }^{40}$, as diferenças verificadas entre rapazes e raparigas nesta habilidade devem-se, fundamentalmente, a variáveis relacionadas com o envolvimento, a diferentes formas de encorajamento, às oportunidades de prática, ao reforço, dentro e fora da escola, visto as raparigas serem educadas de forma diferente dos rapazes e as expectativas criadas diferirem para uns e para outros.

Zaichkowsky $^{47}$, Gallahue ${ }^{10}$, Malina e Bouchard ${ }^{22}$ apontam, como factores determinantes para estas diferenças um conjunto de factores ambientais, como os modelos culturais que se traduzem em oportunidades de prática diferenciada, dependentes dos hábitos de prática motora, 
lúdica e desportiva que determinadas culturas promovem e motivam. Outros factores têm a ver com os processos de aprendizagem vivenciados pelas crianças através da qualidade e quantidade de experiências susceptíveis de lhes garantir o sucesso na aprendizagem e gosto pela prática motora. Quando se observam crianças em situação de actividade livre regista-se uma superioridade, frequência e tempo de utilização de movimentos manipulativos por parte do sexo masculino ${ }^{35}$.

As diferenças entre os géneros nesta habilidade não são apenas restritas ao produto de desempenho, mas também à forma como executam esse movimento (processo). O padrão de lançamento dos rapazes é mais maduro do que o das raparigas, demonstrando uma maior amplitude de movimentos, um passo mais comprido e um movimento de braços mais rápido ${ }^{29}$. Loveall e Nelson ${ }^{16}$ registaram diferenças no padrão de lançar em crianças do jardim-de-infância e esta superioridade registou-se em todas as idades e aumentava à medida que a idade avançava. As acções motoras que explicam as diferenças entre o sexo masculino e o sexo feminino para esta habilidade são diferentes nos dois sexos. Para os rapazes, é fundamentalmente a acção do braço na idade pré-escolar e a acção da perna no $3 .^{\circ}$ grau que explicam a variação do lançamento. Para as raparigas, a variação é explicada pela rotação do tronco na idade pré-escolar e $3 .^{\circ}$ grau e a acção do braço no . $^{\circ}$ grau.

Por outro lado, as raparigas levam mais tempo a passar do $2 .^{\circ}$ para o $3 .^{\circ}$ e do $3 .^{\circ}$ para o $4 .^{\circ}$ estádios de desenvolvimento ${ }^{37}$.

Relativamente à habilidade pontapé, devido à grande variação registada na prestação, a idade não parece ser um bom indicador do desenvolvimento desta habilidade ${ }^{8}$. Estudos realizados com crianças e adultos, com programas direccionados para o desenvolvimento e aprendizagem desta habilidade, os resultados apontam para uma fraca relação entre tempo de prática, fornecimento de feedback e de demonstração ${ }^{36,30}$.
Os rapazes desde muito cedo demonstram frequências superiores às raparigas nas ocorrência das acções motoras pontapear e correr ${ }^{35}$. Os ganhos obtidos na prestação com o decorrer do tempo são superiores nos rapazes, quando foi avaliada a prestação relativamente ao produto de desempenho ${ }^{4}$.

As diferenças verificadas no processo também são grandes. Os rapazes atingem o padrão maduro por volta dos 7 anos, enquanto que as raparigas, o conseguem apenas por volta dos 8,5 anos. Na passagem dos estádios de desenvolvimento, as raparigas levam muito mais tempo na passagem do $2 .^{\circ}$ para o $3 .^{\circ}$ estádio, comparativamente com os rapazes. A passagem do $10^{\circ}$ para o $2 .^{\circ}$ e do $3 .^{\circ}$ para o $4 .^{\circ}$ estádio é idêntica em ambos os sexos. As idades com que atingem cada um dos estádios de desenvolvimento variam, sobretudo no $3 .^{\circ}$ e $4 .^{\circ}$ estádio.

Face ao exposto definimos os seguintes objectivos:

1- Verificar qual o padrão de desenvolvimento nas habilidades: correr, saltar, lançar e pontapear;

2- Verificar se existem diferenças entre os géneros na prestação quantitativa e qualitativa nas habilidades: correr, saltar, lançar e pontapear;

3- Verificar qual a magnitude das diferenças nas habilidades manipulativas e locomotoras, entre o sexo feminino e masculino.

\section{Metodologia}

\section{Amostra}

Participaram no estudo 141 crianças com idades compreendidas entre os 7 e 8 anos, que frequentavam o Ensino Básico $1 .^{\circ} \mathrm{Ciclo}, 1^{\circ} \mathrm{Fase}$, 2..$^{\circ}$ ano pertencentes aos concelhos de Alijó, Régua, Chaves e Valpaços.

A amostra do nosso trabalho é constituída por 141 crianças, 79 do sexo masculino e 62 do sexo feminino, com uma idade média de 91,1 meses. 
Diferenças entre géneros nas habilidades: correr, saltar, lançar e pontapear

Maria Isabel Mourão Carvalhal, José Vasconcelos-Raposo

Quadro 1: Caracterização da amostra por idade (decimal) e sexo.

\begin{tabular}{lccccc} 
& N & Média & $\begin{array}{c}\text { Desvio } \\
\text { Padrão }\end{array}$ & Mínimo & Máximo \\
\hline Sexo masculino & 79 & 7,87 & 0,31 & 7,26 & 8,99 \\
Sexo feminino & 62 & 7,88 & 0,28 & 7,25 & 8,42 \\
Total & 141 & 7,87 & 0,30 & 7,25 & 8,99 \\
\hline
\end{tabular}

\section{Variáveis}

Foi definida como variável independente o género: masculino e feminino.

As variáveis dependentes relativas ao desempenho quantitativo, nas habilidades motoras, fundamentais foi para a corrida em velocidade tempo de corrida (TC), para o salto de impulsão horizontal - distância do salto (DS), para o lançamento de uma bola de ténis por cima da cabeça - distância do lançamento (DL) e para o pontapé de uma bola parada - distância do pontapé (DP).

As variáveis relativas ao desempenho qualitativo para cada uma das habilidades foram os estádios de desenvolvimento (Inicial, Elementar e Maduro) para cada habilidade: correr, saltar, lançar e pontapear.

\section{Instrumentos}

De forma a atingir os objectivos deste trabalho foi necessário utilizar alguns equipamentos.

$\mathrm{Na}$ avaliação das variáveis dependentes, relativamente ao desempenho motor da criança, foram utilizados testes de avaliação do desempenho motor para cada uma das provas, corrida em velocidade, salto de impulsão horizontal sem balanço com os dois pés, lançamento em distância de uma bola de ténis por cima da cabeça e pontapé de uma bola parada.

Para verificar a fiabilidade das medidas obtidas para cada habilidade nos diferentes ensaios, foi utilizado o teste de correlação intra-classe, de acordo com a fórmula: $\mathrm{R}=\left(\mathrm{MS}_{\mathrm{S}}-\mathrm{MS}_{\mathrm{E}}\right) / \mathrm{MS}_{\mathrm{S}} \mathrm{e}$ como podemos observar no quadro $\mathrm{n}^{\circ} 2$, o índice de correlação para cada uma das provas é alto e os valores variam entre 0,98 , para o salto, e 0,99 , para as outras três provas.

Quadro 2: Coeficiente de correlação intra-classe (R) das provas motoras.

\begin{tabular}{lc} 
Provas Motoras & R \\
\hline Corrida & 0,99 \\
Salto & 0,99 \\
Lançamento & 0,99 \\
Pontapé & 0,98 \\
\hline
\end{tabular}

$\mathrm{Na}$ avaliação qualitativa do desempenho da corrida, salto, lançamento e pontapé, foram utilizadas duas câmaras SVHS e um monitor Sony.

Foram também utilizadas as checklist de Gallahue $^{10}$, para cada uma das habilidades. Antes de iniciar o visionamento das habilidades em estudo, o investigador treinou a observação de cada uma das habilidades e classificou-as de acordo com o instrumento seleccionado.

Para verificar o grau de fidelidade intra-observador aplicou o índice de fidelidade de Bellack em dois momentos diferentes, e, após ter obtido índices de fidelidade superiores a $85 \%$, como são recomendados pelo mesmo autor, passou então a avaliar cada uma das habilidades. Tal como se pode observar no quadro $\mathrm{n}^{\circ} 3$, os índices de fidelidade intra-observador variaram entre $85 \%$ para o lançamento e os $95 \%$ para o pontapé, tendo a corrida e o lançamento obtido valores de $88,2 \%$ e $85 \%$ respectivamente. 
Quadro 3: Valores dos Índices de Bellack para as variáveis motoras.

\begin{tabular}{lc} 
Variáveis & Índice de Fidelidade \\
\hline Corrida & $88,2 \%$ \\
Salto & $85 \%$ \\
Lançamento & $95 \%$ \\
Pontapé & $86 \%$ \\
\hline
\end{tabular}

Para comparar a prestação do sexo feminino com a do masculino, nas variáveis contínuas, foi utilizado o teste $t$ de Student, para amostras independentes nas variáveis categóricas, medidas em escalas ordinais foi utilizado o teste de $U$ de Mann-Whitney.

\section{Resultados}

O sexo masculino apresentou índices de prestação a nível quantitativos superiores aos do sexo feminino em todas as provas motoras.

Da leitura dos dados do quadro $\mathrm{n}^{\circ} 4$, podemos verificar que a média (2,1 e 2,3), para o sexo masculino e feminino, respectivamente, indica-nos que o sexo masculino apresenta uma superioridade na prestação da corrida. Tendo em conta os valores de $\mathrm{t}$ e de $\mathrm{p}(\mathrm{t}=5,4 ; \mathrm{p}=0,00)$ registados poderemos concluir que existem diferenças significativas entre os grupos, com superioridade do sexo masculino nesta habilidade motora.

$\mathrm{Na}$ habilidade do salto, a média obtida $(0,9$; e 0,8$)$, respectivamente para o sexo masculino e sexo feminino, indicam-nos diferenças entre os dois grupos. Face ao valor de t e de p $(5,6 ; 0,00)$ obtido, no quadro $\mathrm{n}^{\circ} 4$, podemos concluir que os dois grupos são diferentes.

Através dos valores da média $(12,5$ e 7,6), obtidos, respectivamente, pelo sexo masculino e pelo sexo feminino, podemos verificar as diferenças de prestação na tarefa de lançamento para os dois sexos. No quadro $\mathrm{n}^{\circ} 4$, podemos ler o valor de t e de $\mathrm{p}(8,9 ; 0,00)$, pelo que podemos inferir que essas diferenças são estatisticamente significativas.

Relativamente ao pontapé, os valores da média $(7,9$ e 4,1$)$, obtidos, respectivamente, pelo sexo masculino e feminino, indicam diferenças entre os dois grupos, face ao valor de t e de p (7,9; $0,00)$ registado, podemos concluir que as diferenças são estatisticamente significativas, pelo que podemos dizer que os dois grupos são diferentes.

Quadro 4: Comparação da prestação (produto) em cada variável, de acordo com o sexo, n, média, desvio padrão, mediana valor de $t$ e $p$.

\begin{tabular}{l|cccc|cccccc} 
& \multicolumn{4}{|c|}{ Masculino } & \multicolumn{7}{c}{ Feminino } \\
Variável & n & M & SD & Mediana & n & M & SD & Mediana & t & p \\
\hline Corrida, seg. & 79 & 2,1 & 0,2 & 2,1 & 62 & 2,3 & 0,2 & 2,3 & 5,4 & $0,000^{*}$ \\
Lançamento, m & 79 & 12,5 & 4,0 & 12,4 & 62 & 7,6 & 1,8 & 7,5 & 8,9 & $0,000^{*}$ \\
Salto, m & 79 & 0,9 & 0,2 & 1,0 & 62 & 0,8 & 0,1 & 0,8 & 5,6 & $0,000^{*}$ \\
Pontapé, m & 79 & 7,9 & 3,0 & 7,7 & 62 & 4,1 & 2,4 & 3,5 & 7,9 & $0,000^{*}$ \\
\hline
\end{tabular}

* $p<0,05$

Da análise dos quadros n. ${ }^{\text {s }} 5,6,7$ e 8, verificamos que o estádio de aquisição de cada uma das habilidades não é idêntico para os dois géneros.
No padrão de corrida, $74,5 \%$ da maioria das crianças situa-se no estádio maduro, não se registando qualquer frequência no padrão inicial. Quando comparamos os dois sexos, 
verificamos que a maioria dos rapazes e das raparigas se encontra no padrão maduro, embora com percentagens diferentes. $\mathrm{O}$ sexo masculino apresenta uma percentagem mais elevada, com $86,1 \%$, enquanto que o sexo feminino apresenta apenas $62,9 \%$ no mesmo nível. Face ao valor de U e p $(1881,5 ; 0,001)$ obtido, podemos dizer que existem diferenças estatisticamente significativas entre os géneros no padrão de corrida.

No salto, ambos os sexos se encontram maioritariamente no nível elementar, apresentando o sexo masculino uma percentagem de $69,6 \%$ e o sexo feminino $85,5 \%$.
Regista-se uma pequena percentagem de $(1,3 \%)$ de rapazes no nível inicial, enquanto que as raparigas apresentam uma percentagem de (6,5\%) neste mesmo nível. No nível maduro, o sexo masculino apresenta uma percentagem mais elevada (29,1\%), comparativamente com o sexo feminino $(8,1 \%)$. Face ao valor de U e p $(1850,0$; $0,001)$ registado, podemos inferir que o padrão de execução do pontapé é diferente.

No padrão de lançamento, observamos grandes diferenças na distribuição das percentagens para cada um dos níveis de prestação nos dois grupos.

Quadro 5: Comparação da prestação (processo) da corrida, de acordo com o sexo, frequência, percentagem, valor de U e p.

\begin{tabular}{llcc|cccc}
\multicolumn{3}{c}{ Masculino } & \multicolumn{4}{c}{ Feminino } \\
Variável & Frequência & $\%$ & Frequência & $\%$ & U & $\%$ \\
\hline \multirow{3}{*}{ Corrida } & Inicial & 0 & 0 & 0 & 0 & 1881,5 & $0,001^{*}$ \\
& Elementar & 11 & 13,9 & 11 & 13,9 & & \\
& Maduro & 68 & 86,1 & 68 & 86,1 & \\
& Total & 79 & 100 & 79 & 100 & \\
\hline
\end{tabular}

$* p<0,05$

Quadro 6: Comparação da prestação (processo) do salto, de acordo com o sexo, frequência, percentagem, valor de $U$ e p.

\begin{tabular}{llcc|cccc}
\multicolumn{3}{c}{ Masculino } & \multicolumn{4}{c}{ Feminino } \\
Variável & & Frequência & $\%$ & Frequência & $\%$ & U & $\%$ \\
\hline \multirow{4}{*}{ Salto } & Inicial & 1 & 1,3 & 4 & 6,5 & 1850,0 & $0,001^{*}$ \\
& Elementar & 55 & 69,6 & 53 & 85,5 & & \\
& Maduro & 23 & 29,1 & 5 & 8,1 & \\
& Total & 79 & 100 & 62 & 100 & \\
\hline
\end{tabular}

${ }^{*} p<0,05$

Quadro 7: Comparação da prestação (processo) do lançamento, de acordo com o sexo, frequência, percentagem, valor de U e p.

\begin{tabular}{llcc|cccc}
\multicolumn{4}{c}{ Masculino } & \multicolumn{4}{c}{ Feminino } \\
Variável & & Frequência & $\%$ & Frequência & $\%$ & U & $\%$ \\
\hline \multirow{3}{*}{ Lançamento } & Inicial & 2 & 2,5 & 32 & 51,6 & 895,5 & $0,000^{*}$ \\
& Elementar & 51 & 64,6 & 29 & 46,8 & & \\
& Maduro & 26 & 32,9 & 1 & 1,6 & \\
& Total & 79 & 100 & 62 & 100 & \\
\hline
\end{tabular}

$* p<0,05$ 
Quadro 8: Comparação da prestação (processo) do pontapé, de acordo com o sexo, frequência, percentagem, valor de $U$ e $p$.

\begin{tabular}{|c|c|c|c|c|c|c|c|}
\hline \multicolumn{4}{|c|}{ Masculino } & \multicolumn{4}{|c|}{ Feminino } \\
\hline Variável & & Frequência & $\%$ & Frequência & $\%$ & U & $\%$ \\
\hline \multirow{4}{*}{ Pontapé } & Inicial & 0 & 0 & 2 & 3,2 & 873,5 & $0,000^{*}$ \\
\hline & Elementar & 12 & 15,2 & 47 & 75,8 & & \\
\hline & Maduro & 67 & 84,8 & 13 & 21,0 & & \\
\hline & Total & 79 & 100 & 62 & 100 & & \\
\hline
\end{tabular}

* $p<0,05$

O sexo masculino encontra-se maioritariamente no nível elementar $(64,6 \%)$, enquanto que o sexo feminino $(51,6 \%)$ se encontra no nível inicial. O nível maduro regista uma percentagem de $32,9 \%$ para o sexo masculino, enquanto que o sexo feminino apresenta apenas uma percentagem de 1,6\% neste mesmo nível. Face ao valor de U e p $(895,5 ; 0,000)$ registado, as diferenças são significativas, pelo que podemos concluir que os dois grupos apresentam um padrão de lançamento diferente.

No pontapé, a distribuição pelos três níveis de prestação, nos dois sexos, também não é igual. O sexo masculino encontra-se, maioritariamente, no nível maduro (84,8\%), enquanto que o sexo feminino se encontra no nível elementar $(75,8 \%)$. Não há rapazes no nível inicial, ao contrário das raparigas, cujo valor percentual, nesse nível, é de $3,2 \%$. No nível maduro, as raparigas apresentam uma percentagem de $21 \%$. Face ao valor de U e p $(873,5 ; 0,000)$ registado, podemos inferir que o padrão de execução do pontapé é diferente.

\section{Discussão}

Quando foram comparados rapazes e raparigas nas tarefas corrida, lançamento, salto e pontapé, tarefas consideradas grosseiras registaram-se diferenças significativas entre os géneros, relativamente ao produto de desempenho, favoráveis ao sexo masculino. Estes resultados vão ao encontro dos estudos efectuados neste âmbito como os de Mline et al. ${ }^{24}$, Morris, et al. ${ }^{25}$, Benefice e Malina ${ }^{1}$, na corrida; Mline et al. ${ }^{24}$, Morris et al..$^{25}$
Benefice e Malina ${ }^{1}$ no salto; Mourão-Carvalhal ${ }^{27}$, Halverson $^{13}$, Benefice e Malina ${ }^{1}$ no lançamento Dias e Nascimento5; Van Beurden et al. ${ }^{44}$; Mourão-Carvalhal ${ }^{28}$, no pontapé DeOreo e Keogh ${ }^{4}$.

As diferenças entre os dois sexos não se registam apenas no produto de desempenho, mas também no processo de desempenho das tarefas, isto é, a forma como se lança é determinante na distância alcançada pela bola, tal como demonstram os resultados de Wickstrom ${ }^{45}$ e Ulrich ${ }^{43}$, que verificaram uma relação positiva, mas não directa, entre a forma e o produto de desempenho. Segundo Ulrich ${ }^{43}$, à medida que se sobe na hierarquia dos estádios de desenvolvimento das habilidades motoras fundamentais, são atingidas execuções mais eficientes.

Dado que se tinham registado diferenças entre os sexos no produto da prestação, era de esperar a existência de diferenças na forma de desempenho. As maiores diferenças, relativamente ao produto, verificaram-se nas habilidades do lançamento e do pontapé, seguidas do salto e da corrida. Numa análise do processo de desempenho, também se registaram diferenças, em ambos os sexos, nas mesmas habilidades. A percentagem de raparigas que atinge o estádio maduro, $0,7 \%$, no lançamento é significativamente inferior à percentagem dos rapazes, $18,4 \%$. No pontapé, $47,5 \%$ dos rapazes atinge o estádio maduro, enquanto que apenas $9,2 \%$ das raparigas atingem esse estádio. No salto, 16,3\% dos rapazes 3,5\% das raparigas atingem o estádio maduro. Na corrida, $48,9 \%$ dos rapazes e $27,7 \%$ das raparigas atingem o estádio maduro. 
Teoricamente, de acordo com o modelo de Gallahue $^{10}$ (1989), seria de esperar, que com esta idade, a maioria das crianças estivessem já no estádio maduro das habilidades estudadas, dado que este estádio é atingido por volta dos 6,7 anos de idade. Os nossos resultados confirmam os de Seefeldt e Haubenstricker ${ }^{38}$. Os autores verificaram que $40 \%$ das crianças com idades compreendidas entre os 6 e 7 anos ainda não tinham atingido o estádio maduro de grande parte dos movimentos fundamentais. Malina e Bouchard $^{22}$ explicam estes resultados pela falta de coordenação motora, devida à imaturação do SNC, o que impede uma maior eficácia na prestação dos movimentos. Espenshade e Eckert ${ }^{9}$ registaram, também, diferenças entre os sexos na habilidade de saltar. O sexo masculino atingiu mais cedo o padrão maduro do salto. Também Seefeld $t^{39}$ registou diferenças entre os sexos no desempenho do salto, com $60 \%$ do sexo masculino a atingirem o nível de desenvolvimento do padrão do salto com a idade de 9 anos e meio e o sexo feminino com a idade de 10 anos. Nelson et al. ${ }^{29}$, Seefeldt e Haubenstricker ${ }^{37}$ regis- $^{-}$ taram diferenças entre os dois sexos na forma de desempenho do lançamento. No estudo de Seefeldt ${ }^{37} 60 \%$ dos rapazes atingiram o lançamento com a idade de 5 anos e $60 \%$ das raparigas com a idade de 8 anos.

De acordo com os dados de Seefeldt ${ }^{37}, 60 \%$ dos rapazes atinge o padrão maduro no pontapé por volta dos 7 anos e $60 \%$ das raparigas por volta dos 8 anos.

Tal como refere Gallahue ${ }^{10}$ o estádio elementar destas habilidades, característico dos 3 e dos 5 anos, é atingido fundamentalmente pelos efeitos de maturação. Mas a maturação biológica, por si só, não é suficiente para transformar as capacidades em habilidades e estas, por sua vez, em técnicas específicas de cada uma das modalidades desportivas. O papel do envolvimento através de uma prática/vivência estimulada, organizada e intencional é fundamental no desenvolvimento de esquemas plásticos e fle- xíveis, que se vão desenvolvendo e adaptando com maior eficácia aos estímulos fornecidos pelo meio envolvente.

É por isso que a maioria dos adultos se encontra neste estádio (elementar) em algumas das habilidades fundamentais, como por exemplo, o lançar e o receber, visto não terem tido oportunidades de prática que permitissem a sua evolução para estádios de desenvolvimento mais avançados. Por outro lado, os mesmos autores referem também que o fracasso e o insucesso nestas habilidades vai comprometer o sucesso e a aquisição dos movimentos especializados e, mais tarde, a participação nos desportos de competição, uma vez que estas habilidades são as habilidades base, praticadas na maioria dos desportos, só que mais refinadas tecnicamente. Por esta razão, Seefeld ${ }^{37}$, realçando a importância da prática e da vivência deste tipo de movimentos nestas idades, introduziu, no seu modelo de desenvolvimento, uma barreira, que denominou de barreira de eficiência, que precede a dos movimentos especializados. Para que esta barreira seja ultrapassada, é necessário que a criança tenha oportunidades de prática e de vivência de todos os movimentos fundamentais, pois encontra-se na fase óptima para aprender. Seefeldt e Haubenstricker ${ }^{37}$ consideram que esta fase óptima se deve chamar de período sensível e não crítico; ao contrário do termo crítico, que significa está dependente, fundamentalmente, da maturação biológica, sensível implica que a aprendizagem ocorre com maior eficácia num determinado período no ciclo de vida, embora possa ser atingida mais tarde.

De todas as tarefas observadas, aquela onde se registou uma maior magnitude de diferenças entre os sexos, foi, sem dúvida, o lançamento, seguida do pontapé, do salto e, por último, da corrida. As tarefas onde se registaram maiores diferenças entre os sexos foram as provas que exigiam a manipulação de objectos (bolas) com as mãos, como é o caso do lançamento, ou com o pé, como é exemplo o pontapé. Estes tipos de habilidades são exigentes quanto ao tipo de espaços necessários para a sua prática. Isto é, 
necessitam de espaços amplos, no exterior para uma melhor experimentação e vivência de acções motoras com bola, desde o lançar, receber, pontapear, ou a combinação de várias acções, como correr, lançar, entre outras. A superioridade do sexo masculino em todas as provas pode ser explicada com base na forma como os dois sexos passam o seu tempo livre. Se tivermos em consideração os estudos sobre a actividade lúdica efectuados na infância, verificamos que o comportamento dos dois sexos é diferente, revelando o sexo masculino maior dinamismo, competitividade e agressividade nas suas brincadeiras, enquanto que o sexo feminino prefere actividades mais sedentárias e com pouca utilização de bolas. De facto, esta forma de brincar do sexo masculino exige espaços mais amplos capazes de permitirem o desenvolvimento de brincadeiras mais dinâmicas, como é o caso das actividades de manipulação de bola, ao contrário do sexo feminino, para quem basta um espaço fechado e pequeno para as suas actividades menos dinâmicas, mas mais interactivas verbalmente.

Uma vez que se regista uma inferioridade nestas habilidades, quer a nível do processo, quer do produto, por parte do sexo feminino, a sua participação nos desportos especializados está comprometida, pelo insucesso e também pelas pressões sociais, nomeadamente as familiares, que vai ter que vencer. Podemos dizer que se entra num ciclo vicioso. A inferioridade registada nestas habilidades por parte do sexo feminino, devido à falta de oportunidades de prática, de encorajamento, e à forma como o seu quarto é equipado, aos brinquedos, (bonecas) para os quartos das raparigas propiciando jogos mais estáticos e comportamentos mais verbais do que motores, enquanto que os dos rapazes têm brinquedos mais complexos (bolas e carros), com componentes móveis, promovendo uma actividade motora mais dinâmica, e em espaços mais amplos.

Também o papel esperado para o sexo feminino e o tipo de brincadeiras que experimenta, contribuirá para que se pense que o sexo feminino apresente desempenhos inferiores aos do sexo masculino, por razões de natureza genética. Esta noção errada, põe de lado todos os processos de enculturação ${ }^{32}$ diferenciados que o sexo feminino sofre ao longo da infância na aprendizagem do seu género sexual, e que têm uma continuidade pela vida fora. Esta inferioridade na prestação motora das habilidades fundamentais, nestas idades, vai comprometer seriamente a sua participação nas actividades desportivas durante a última infância e adolescência, bem como no desenvolvimento de hábitos de actividade física ao longo da vida, acarretando implicações a nível da saúde, nomeadamente nas doenças hipocinéticas, tão características do sexo feminino.

Todos estes aspectos vão levar a que os pais, educadores e a sociedade em geral confirmem a ideia de que o sexo feminino é, realmente, inferior ao sexo masculino no desempenho motor, de uma forma global.

Thomas e Thomas ${ }^{40}$ são de opinião que as diferenças entre os sexos, a nível da actividade motora, durante a infância são induzidas pelo envolvimento; depois da puberdade, são fruto de uma interacção entre envolvimento e biologia.

A explicação do desenvolvimento humano, e, do motor em particular, não se pode centrar em modelos ou teorias que procuram interpretar comportamentos numa única dimensão. O Ser Humano não é uma soma de diferentes partes, independentes umas das outras, mas funciona como um todo, um conjunto, onde todas as componentes se interpenetram e que, como afirmam Shweder ${ }^{38}$ e Raposo ${ }^{32}$ age intencionalmente num determinado contexto que é, por sua vez, igualmente intencional.

\section{Correspondência}

Universidade Trás-os-Montes e Alto Douro

Avenida Dr. Manuel Cardona

CIFOP - Departamento Desporto

5000 Vila Real

E-mail: j.vasconcelos.raposo@gmail.com 


\section{Referências}

1. Benefice, E. e Malina, R. (1996). Body size, body composition, and motor performances of mild-to-moderately undernourished Senegalese children. Annals Of Human Biology, 23, 4, 307-321.

2. Branta, C.; Haubenstricker, J. e Seefeldt, V. (1984). Age changes in motor skills during childhood and adolescence. Exercise Sport Science Review, 12, 467-521.

3. Burton, A. W., Dancisak, M. e Rodgerson, R. W. (2000). Effect of age, sex, and distance on throwing accuracy and velocity.

4. DeOreo, K, L. e Keogh, J. (1980). Performance of Fundamental Motor Tasks. In: B. Corbin (Eds). "A text of motor development", pp.76-91 (2 $2^{\mathrm{a}}$ Ed.). Dubuque, IA: Wm.C.Brown.

5. Dias, M. A. e Nascimento M. (2003). Educação e aptidão física no $1 .^{\circ} \mathrm{CEB}$ : Um contributo para a sua caracterização. Horizonte 105: 3-11.

6. Eaton, W. O. e Yu, P. A. (1989). Are sex differences in child motor activity level a function of sex differences in maturational status? Child Development, 60, 1005-1011.

7. Eckert, H. M. (1993). Desenvolvimento motor ( $3^{a}$ Ed.). São Paulo: Manole Ltd ${ }^{a}$.

8. Elliot, B. C., Bloomfield, J. e Davies, C. M. (1980). Development of the punt kick: A cinematographic analysis. Journal of Human Movement Studies, 6, 142-50.

9. Espenshade, E. e Eckert, H. (1980) Motor development. ( $2^{\mathrm{a}}$ Ed.) Ohio: Charles E. Merrill Publishing Company.

10. Gallahue, D. (1989). Understanding motor development: Infants, children, adolescents. (2 ${ }^{\mathrm{a}} \mathrm{Ed}$.) Indianapolis: Benchmark Press, Inc.

11. Greendorfer, S. L. (1977). Role of socializing agents in female sport involvement. Research Quarterly, 48, 304-310. Champaign Ill: Human Kinetics.

12. Halverson, L. E., Roberton, M. A. e Langendorfer, S. (1982) Development of the overarm throw: Movement and ball velocity changes by seventh grade. Research Quarterly for Exercise and Sport, 53, 198-205.

13. Halverson, L., Roberton, M., Safrit, M. e Roberts, T. (1977). Effects of guided practice on overhand-throw ball velocities of kindergarten children. Research Quarterly For Exercise and Sport, 48, 311-318.

14. Hardin, D. e Garcia, M. (1982). Diagnostic performance tests for elementary children (grades 1-4). Physical Education Recreation Dance, 53, 48-52.

15. Haubenstricker, J. L. e Sapp, M. M. (1980) A longitudinal look at physical growth and motor performance: Implications for elementary and middle school activity programs. Paper presented at the Annual Convention of the American Alliance for Health, Physical Education, Recreation and dance, Michigan.

16. Haubenstricker, J. L. e Seefeldt, V. (1986). Acquisition of motor skills during childhood. In V. Seefeldt (Eds.), Physical activity and well-being, pp. 41-102. Reston, VA: American Alliance for Health, Physical Education, Recreation and Dance.

17. Haywood, K. M. (1986). Life span motor development. Champaign, Il: Human Kinetics.

18. Keogh, J. F. e Sugden, D. (1985). Movement skill development. New York: MacMillan.

19. Loveall, K.; Nelson, K. (1992). Gender differences in children's throwing performance across Age: Biological and environmental influences. Research Quarterly For Exercise and Sport, 63, Supplement $\mathrm{N}^{\mathrm{o}} 1$.

20. Maccoby, E. \& Jacklin, C. (1974). The psychology of sex differences. Stanford, CA: Stanford University Press.

21. Malina, R. (1984). Human Growth, maturation and regular Physical Activity. In Richard A. Boileau (Eds.) Advances in Pediatric Sport Sciences, pp. 59-83, (Vol.1). Champaign Ill: Human Kinetics. 
22. Malina, R. M. \& Bouchard, C. (1991). Growth maturation, and physical activity. Champaign, Ill: Human Kinetics.

23. Malina, R. M. (1987). Biocultural determinants of motor development. Paper presented at Conference of the Association International des Ecolles Universitaires d'Education Physique. Lisboa: Portugal.

24. Mline, C., Seefeldt, V. \& Reuchlein, Ph. (1976). Relationship between grade, sex, race and motor performance in young children. Research Quarterly For Exercise and Sport, 47, 4, 726-730.

25. Morris, A. M., Williams, J. M., Atwater, A. E. e Wilmore, J. H. (1982). Age and sex differences in motor performance of 3 through 6 year old children. Research Quarterly For Exercise and Sport, 53, 3, 214-221.

26. Mourão-Carvalhal, I. (2007). Desenvolvimento e aprendizagem do lançamento em distância por cima do ombro. In: J. Barreiros (Ed.). Desenvolvimento Motor da Criança. Cruz Quebrada: F. M. H. UTL, pp. 309-315.

27. Mourão-Carvalhal, I. M. (1991). Contributo para o estudo do desenvolvimento motor Infantil, crianças da cidade de Vila Real. Trabalho realizado no âmbito do Mestrado, não publicado.

28. Mourão-Carvalhal, I. M. (1995). Maturação e aprendizagem do padrão de corrida em crianças de 2,3 anos de Idade. Tese de Mestrado. Lisboa: FMH, UTL.

29. Nelson, J. K., Thomas, J. R. e Nelson, K. R. (1986). Gender differences in children's throwing performance: Biology and environment. Research Quarterly For Exercise And Sport, 57, 280-287.

30. Neto, C. (1987). Motricidade e Desenvolvimento: estudo do comportamento de crianças de 5-6 anos relativo à influência de diferentes estímulos pedagógicos na aquisição de habilidades fundamentais de manipulação. Tese de Doutoramento. Lisboa: UTL/FMH.
31. Pruetz, J. D. e Bertolani, P. (2007). Savanna chimpanzees pan troglodytes verus, hunt with tools. Current Biology, 17, 412-417.

32. Raposo, J. V. (1996). Psicologia do desporto: Passado, presente e futuro. Vila Real: UTAD Série Técnico Científica.

33. Reuchlein, P. e Vogel, P. (1985). Motor performance and physical fitness status of regular and special education students. In J. E. Clarck e J. H. Humphrey (Eds.) Motor development: Current selected research, pp. 147-165, (Vol.1). Princeton, NJ: Princeton Book Company.

34. Roberton, M. A., Halverson, L. E. e Williams, K. (1979) Longitudinal changes in children's overhand throw ball velocities. Research Quarterly, 50, 256-264.

35. Rodeia, A. (1985) Estudo observacional do comportamento motor infantil na utilização de materiais portáteis A Bola. Ludens, 9, 18-24.

36. Scott, M. A. e William, M. A. (2003). The co-ordination of kicking techiniques in children. In G. Savelsberggh; K. Davids; J. van der Kamp, e S. J. Bennett (Eds). Development of movement Co-ordination in children Aplications in the fields of ergonomics, health sciences and sport (pp. 241-250). London: Routledge.

37. Seefeldt, V. e Haubenstricker, J. (1982). Patterns, phases or stages: An analytical model for the study of developmental movement. In J. A S. Kelso e J. E. Clarck (Eds). The development of movement control and coordinatio" (pp. 309-318). New York: John Wiley \& Sons.

38. Shweder, R. (1984). Preview: A Colloquy of culture theories. In Robert A. Shweder e Robert A. Levine (Eds.) Culture Theory: Essays on Mind, Self and Emotions. New York: Cambridge University Press.

39. Thomas, J. R. e French, K. E. (1985). Gender differences across age in motor performance: A meta-analysis. Psychological Bulletin, 98, 260-282. 
40. Thomas, J. R. e Thomas, K. T. (1988). Development of gender differences in physical activity. Quest, 40, 219-229.

41. Thomas, J. R. (2000). Children's control, learning, and performance of motor skills. Research Quarterly for Exercise and Sport, 71, 1-9.

42. Thomas, J. R., Thomas, K. T. e Gallagher, J. D. (1993) Developmental considerations In skill acquisition. In Robert Singer, Milledge Murphey, L. Keith Tennant (Eds). Handbook of Research on Sport Psychology (pp.73-105). New York: Macmillan Publishing Company.

43. Ulrich, B. (1987). Developmental perspectives of motor skill performance in children. In D. Gould, D. e M. Weiss (Eds). Advances in
Pediatric Sport Sciences (pp. 167-187), (Vol Two). Champaign Ill: Human Kinetics.

44. Van Beurden, E., Zask, A., Barnett, L. M. e Dietrich, U. C. (2002). Fundamental movement skills-how do primary school children perform? The move it groove it program in rural Australia. Journal of Science and Medicine in Sport, 5: 244-252.

45. Wickstrom, R. (1983). Patrones motores básicos. Madrid: Alianza Editorial.

46. Williams, N. (1983). Perceptual and motor development. New Jersey: Prentice-Hall, Inc., Englewood Cliffs.

47. Zaichkowsky, L. D., Zaichkowsky, L. B. e Martinek, Th. J. (1980). Growth and development the child and physical activity. London: Mosby Company. 